\title{
NEUMANN PROBLEMS WITH INDEFINITE AND UNBOUNDED POTENTIAL AND CONCAVE TERMS
}

\author{
NIKOLAOS S. PAPAGEORGIOU AND VICENŢIU D. RĂDULESCU
}

(Communicated by Catherine Sulem)

\begin{abstract}
We consider a semilinear parametric Neumann problem driven by the negative Laplacian plus an indefinite and unbounded potential. The reaction is asymptotically linear and exhibits a negative concave term near the origin. Using variational methods together with truncation and perturbation techniques and critical groups, we show that for all small values of the parameter the problem has at least five nontrivial solutions, four of which have constant sign.
\end{abstract}

\section{INTRODUCTION}

Let $\Omega \subseteq \mathbb{R}^{N}$ be a bounded domain with a $C^{2}$-boundary $\partial \Omega$. In this paper, we study the following parametric Neumann problem:

$\left(P_{\lambda}\right)-\Delta u(z)+\beta(z) u(z)=f(z, u(z))-\lambda|u(z)|^{q-2} u(z)$ in $\Omega, \quad \frac{\partial u}{\partial n}=0$ on $\partial \Omega$.

In the boundary condition $n(\cdot)$ denotes the outward unit normal on $\partial \Omega$. Also, in $\left(P_{\lambda}\right)$ the potential function $\beta(\cdot)$ need not be bounded and may change sign (indefinite potential). So, the differential operator $u \mapsto-\Delta u+\beta u$ is not in general coercive. In the reaction (the right-hand side of problem $\left(P_{\lambda}\right)$ ), the function $f(z, x)$ is a Carathéodory function (that is, for every $x \in \mathbb{R}, z \mapsto f(z, x)$ is measurable and for a.a. $z \in \Omega, x \mapsto f(z, x)$ is continuous). We assume that for a.a. $z \in \Omega, f(z, \cdot)$ exhibits linear growth near $\pm \infty$ and interacts with the principal eigenvalue $\hat{\lambda}_{1}(\beta)$ of the differential operator $u \mapsto-\Delta u+\beta u$ with Neumann boundary condition. In the other part of the reaction, namely the term $-\lambda|x|^{q-2} x, \lambda>0$ is a parameter and $q \in(1,2)$. So, this is a concave term which enters in the reaction with a negative sign. Hence we are dealing with an equation resonant at $\pm \infty$ with respect to $\hat{\lambda}_{1}(\beta)$.

Semilinear elliptic equations with concave contributions in the reaction were first considered by Ambrosetti, Brezis and Cerami [2, who studied problems in which the concave term enters with a positive sign and the perturbation $f(z, x)=f(x)$ is superlinear (problems with concave-convex nonlinearities). This leads to a different geometry for the problem. Problems with a negative concave term in the reaction were examined by Perera [13] and de Paiva and Massa [5]. Both deal with Dirichlet equations with no potential term $\beta u$ (that is, $\beta \equiv 0$ ) and no resonance is permitted.

Recently the authors (see Papageorgiou and Rădulescu [11]) examined nonparametric semilinear Neumann problems with an indefinite and unbounded potential,

Received by the editors February 3, 2014 and, in revised form, August 2, 2014.

2010 Mathematics Subject Classification. Primary 35J20; Secondary 35J60, 58E05.

Key words and phrases. Concave nonlinearity, indefinite and unbounded potential, resonance, Harnack inequality, regularity theory, local minimizers. 
with no concave terms and with a crossing nonlinearity. Analogous problems driven by the $p$-Laplacian were studied by Mugnai and Papageorgiou [9] and Papageorgiou and Rădulescu [12].

\section{MathematicAl BACKGround}

Let $X$ be a Banach space and $X^{*}$ its topological dual. By $\langle\cdot, \cdot\rangle$ we denote the duality brackets for the pair $\left(X^{*}, X\right)$. Let $\varphi \in C^{1}(X)$. We say that $\varphi$ satisfies the "Palais-Smale condition" ("PS-condition" for short) if the following property holds:

"Every sequence $\left\{u_{n}\right\}_{n \geqslant 1} \subseteq X$ such that $\left\{\varphi\left(u_{n}\right)\right\}_{n \geqslant 1} \subseteq \mathbb{R}$ is bounded and $\varphi^{\prime}\left(u_{n}\right)$ $\rightarrow 0$ in $X^{*}$ as $n \rightarrow \infty$ admits a strongly convergent subsequence."

This compactness type condition on $\varphi$ compensates for the fact that the ambient space $X$ need not be locally compact (when $X$ is infinite dimensional) and is the main tool in proving a deformation theorem. This deformation theorem leads to a minimax theory for the critical values of $\varphi$. One of the main results in that theory is the so-called mountain pass theorem; see Ambrosetti and Rabinowitz 3 .

In our analysis of problem $\left(P_{\lambda}\right)$ in addition to the Sobolev space $H^{1}(\Omega)$, we will also use the Banach space $C^{1}(\bar{\Omega})$. This is an ordered Banach space with positive cone given by

$$
C_{+}=\left\{u \in C^{1}(\bar{\Omega}): u(z) \geqslant 0 \text { for all } z \in \bar{\Omega}\right\} .
$$

This cone has a nonempty interior given by

$$
\text { int } C_{+}=\left\{u \in C_{+}: u(z)>0 \text { for all } z \in \bar{\Omega}\right\} .
$$

Consider a Carathéodory function $f_{0}: \Omega \times \mathbb{R} \rightarrow \mathbb{R}$ which satisfies subcritical growth with respect to the $x \in \mathbb{R}$ variable, that is,

$$
\left|f_{0}(z, x)\right| \leqslant a(z)\left(a+|x|^{r-1}\right) \text { for a.a. } z \in \Omega \text {, all } x \in \mathbb{R},
$$

with $a \in L^{s}(\Omega), s>\frac{N}{2}$ if $N=3, s>1$ if $N=2$, and $s=1$ if $N=1$ and $r \in\left(1,2^{*}\right)$, where $2^{*}$ is the critical Sobolev exponent, namely

$$
2^{*}=\left\{\begin{array}{cl}
\frac{2 N}{N-2} & \text { if } N>2, \\
+\infty & \text { if } N=1,2 .
\end{array}\right.
$$

Let $F_{0}(z, x)=\int_{0}^{x} f_{0}(z, \tau) d \tau$ and consider the $C^{1}$-functional $\varphi_{0}: H^{1}(\Omega) \rightarrow \mathbb{R}$ defined by

$$
\varphi_{0}(u)=\frac{1}{2}\|D u\|_{2}^{2}-\int_{\Omega} F_{0}(z, u(z)) d z \text { for all } u \in H^{1}(\Omega) .
$$

The next result is essentially a particular case of Theorem 2.1 of Motreanu and Papageorgiou [8], and its proof uses the regularity result of Wang [15].

Proposition 1. Assume that $u_{0} \in H^{1}(\Omega)$ is a local $C^{1}(\bar{\Omega})$-minimizer of $\varphi_{0}$; that is, there exists $\varrho_{0}>0$ such that

$$
\varphi_{0}\left(u_{0}\right) \leqslant \varphi_{0}\left(u_{0}+h\right) \text { for all } h \in C^{1}(\bar{\Omega}) \text { with }\|h\|_{C^{1}(\bar{\Omega})} \leqslant \varrho_{0} .
$$

Then $u_{0} \in C^{1}(\bar{\Omega})$ and it is a local $H^{1}(\Omega)$-minimizer of $\varphi_{0}$; that is, there exists $\varrho_{1}>0$ such that

$$
\varphi_{0}\left(u_{0}\right) \leqslant \varphi_{0}\left(u_{0}+h\right) \text { for all } u \in H^{1}(\Omega) \text {, with }\|u\| \leqslant \varrho_{1} .
$$


Remark 1 . Here and in the sequel, by $\|\cdot\|$ we denote the norm of the Sobolev space $H^{1}(\Omega)$. Therefore

$$
\|u\|=\left(\|u\|_{2}^{2}+\|D u\|_{2}^{2}\right)^{1 / 2} \text { for all } u \in H^{1}(\Omega) .
$$

Also, we should mention that the first such result relating local minimizers was proved by Brezis and Nirenberg [4] for functionals defined on $H_{0}^{1}(\Omega)$.

In what follows by $\tau: H^{1}(\Omega) \rightarrow \mathbb{R}$ we denote the $C^{1}$-functional defined by

$$
\tau(u)=\|D u\|_{2}^{2}+\int_{\Omega} \beta(z) u(z)^{2} d z \text { for all } u \in H^{1}(\Omega) .
$$

We assume that $\beta \in L^{s}(\Omega)$ with $s>\frac{N}{2}$ and also assume that $N \geqslant 3$, since for $N=1,2$, the situation is straightforward due to the Sobolev embedding theorem. Let $s^{\prime}$ denote the conjugate exponent of $s$ (that is, $\frac{1}{s}+\frac{1}{s^{\prime}}=1$ ). We have $s^{\prime}<\frac{N}{N-2}$ and so $2 s^{\prime}<2^{*}$. Then the Sobolev embedding theorem implies that $u^{2} \in L^{s^{\prime}}(\Omega)$. Using the Hölder inequality, we have

$$
\left|\int_{\Omega} \beta(z) u^{2} d z\right| \leqslant\|\beta\|_{s}\left(\int_{\Omega} u^{2 s^{\prime}} d z\right)^{\frac{1}{s^{\prime}}}=\|\beta\|_{s}\|u\|_{2 s^{\prime}}^{2} .
$$

We know that

$$
H^{1}(\Omega) \hookrightarrow L^{2 s^{\prime}}(\Omega) \hookrightarrow L^{2}(\Omega),
$$

and by the Sobolev embedding theorem, the first embedding is compact. So, invoking Ehrling's inequality (see, for example, Papageorgiou and Kyritsi [10, p. 698]), given $\varepsilon>0$, we can find $c(\varepsilon)>0$ such that

$$
\|u\|_{2 s^{\prime}}^{2} \leqslant \varepsilon\|u\|^{2}+c(\varepsilon)\|u\|_{2}^{2} \text { for all } u \in H^{1}(\Omega) .
$$

From (11) and (2), we see that for all $u \in H^{1}(\Omega)$, we have

$$
\begin{gathered}
\|D u\|_{2}^{2}+\|u\|_{2}^{2}-\int_{\Omega} \beta(z) u^{2} d z \leqslant\|D u\|_{2}^{2}+\|u\|_{2}^{2}+\varepsilon\|\beta\|_{s}\|u\|^{2}+c(\varepsilon)\|\beta\|_{s}\|u\|_{2}^{2} \\
\Rightarrow\left(1-\varepsilon\|\beta\|_{s}\right)\|u\|^{2} \leqslant \tau(u)+\left(1+c(\varepsilon)\|\beta\|_{s}\right)\|u\|_{2}^{2} .
\end{gathered}
$$

If we choose $\varepsilon \in\left(0, \frac{1}{\|\beta\|_{s}}\right)$, then from (3) it follows that

$$
\hat{c}\|u\|^{2} \leqslant \tau(u)+\gamma\|u\|_{2}^{2} \text { for all } u \in H^{1}(\Omega), \text { with } \hat{c} \in(0,1), \gamma>0 .
$$

We consider the following linear eigenvalue problem:

$$
-\Delta u(z)+\beta(z) u(z)=\hat{\lambda} u(z) \text { in } \Omega, \quad \frac{\partial u}{\partial n}=0 \text { on } \partial \Omega .
$$

From Papageorgiou and Rădulescu [11, we know that problem (5) admits a sequence $\left\{\hat{\lambda}_{k}(\beta)\right\}_{k \geqslant 1}$ of eigenvalues such that $\hat{\lambda}_{k}(\beta) \rightarrow+\infty$ as $k \rightarrow \infty$ and the corresponding eigenfunctions belong in $C^{1}(\bar{\Omega})$ if $s>N$ and form orthogonal bases for the separable Hilbert spaces $L^{2}(\Omega)$ and $H^{1}(\Omega)$. The principal eigenvalue $\hat{\lambda}_{1}(\beta)$ is simple (that is, the corresponding eigenspace is one dimensional) and admits the following variational characterization:

$$
\hat{\lambda}_{1}(\beta)=\inf \left[\frac{\tau(u)}{\|u\|_{2}^{2}}: u \in H^{1}(\Omega), u \neq 0\right] .
$$

The infimum is realized on the corresponding one-dimensional eigenspace. Let $\hat{u}_{1}(\beta) \in C^{1}(\bar{\Omega})$ be the $L^{2}$-normalized (that is, $\left\|\hat{u}_{1}(\beta)\right\|_{2}=1$ ) principal eigenfunction. From (66) we see that it does not change sign and we choose it to be positive, 
that is, $\hat{u}_{1}(\beta) \in C_{+}$. Moreover, from Harnack's inequality (see Pucci and Serrin [14, p. 163]), we obtain $\hat{u}_{1}(\beta)(z)>0$ for all $z \in \Omega$. In fact if we assume that $\beta^{+} \in L^{\infty}(\Omega)$, using the maximum principle (see Pucci and Serrin [14, p. 120]), we can conclude that $\hat{u}_{1}(\beta) \in$ int $C_{+}$. We mention that $\hat{\lambda}_{1}(\beta)$ is the only eigenvalue with eigenfunctions of constant sign. All the other eigenvalues have nodal (sign changing) eigenfunctions. By $E\left(\hat{\lambda}_{k}(\beta)\right)$ we denote the eigenspace corresponding to the eigenvalue $\hat{\lambda}_{k}(\beta)$. We have the following orthogonal direct sum decomposition:

$$
H^{1}(\Omega)=\overline{\oplus_{k \geqslant 1} E\left(\hat{\lambda}_{k}(\beta)\right)} .
$$

The nonprincipal eigenvalues have the following variational characterizations:

$$
\begin{aligned}
\hat{\lambda}_{m}(\beta) & =\inf \left[\frac{\tau(u)}{\|u\|_{2}^{2}}: u \in \bigoplus_{k \geqslant m} E\left(\hat{\lambda}_{k}(\beta)\right)\right] \\
& =\sup \left[\frac{\tau(u)}{\|u\|_{2}^{2}}: u \in \bigoplus_{k=1}^{m} E\left(\hat{\lambda}_{k}(\beta)\right)\right], m \geqslant 2 .
\end{aligned}
$$

Both the infimum and the supremum are realized on $E\left(\hat{\lambda}_{m}(\beta)\right)$.

Finally, we mention that the eigenspaces $E\left(\hat{\lambda}_{k}(\beta)\right), k \geqslant 1$, have the unique continuation property; namely, if $u \in E\left(\hat{\lambda}_{k}(\beta)\right)$ and $u$ vanishes on a set of positive Lebesgue measure, then $u \equiv 0$.

The following proposition is an easy consequence of the spectral properties of $-\Delta u+\beta(z) u$ described above. See also Papageorgiou and Rădulescu [1] (Proposition 2.3) for $(a)$ and Gasinski and Papageorgiou [6] for $(b),(c)$.

Proposition 2. (a) If $\vartheta \in L^{\infty}(\Omega), \vartheta(z) \leqslant \hat{\lambda}_{1}(\beta)$ a.e. in $\Omega, \vartheta \neq \hat{\lambda}_{1}(\beta)$, then there exists $c_{0}>0$ such that

$$
\tau(u)-\int_{\Omega} \vartheta(z) u^{2} d z \geqslant c_{0}\|u\|^{2} \text { for all } u \in H^{1}(\Omega) .
$$

(b) If $\eta \in L^{\infty}(\Omega), \eta(z) \leqslant \hat{\lambda}_{m+1}(\beta)$ a.e. in $\Omega, \eta \neq \hat{\lambda}_{m+1}(\beta)$, then there exists $\tilde{\xi}_{0}>0$ such that

$$
\tau(u)-\int_{\Omega} \eta(z) u^{2} d z \geqslant \tilde{\xi}_{0}\|u\|^{2} \text { for all } u \in \bigoplus_{k \geqslant m+1} E\left(\hat{\lambda}_{k}(\beta)\right) .
$$

(c) If $\eta \in L^{\infty}(\Omega), \eta(z) \geqslant \hat{\lambda}_{m}(\beta)$ a.e. in $\Omega, \eta \neq \hat{\lambda}_{m}(\beta)$, then there exists $\hat{\xi}_{0}>0$ such that

$$
\tau(u)-\int_{\Omega} \eta(z) u^{2} d z \leqslant-\hat{\xi}_{0}\|u\|^{2} \text { for all } u \in \bigoplus_{k=1}^{m} E\left(\hat{\lambda}_{k}(\beta)\right) .
$$

Let $\left(Y_{1}, Y_{2}\right)$ be a topological pair such that $Y_{2} \subseteq Y_{1} \subseteq X$. For every integer $k \geqslant 0$ we denote by $H_{k}\left(Y_{1}, Y_{2}\right)$ the $k$ th relative singular homology group with integer coefficients for the pair $\left(Y_{1}, Y_{2}\right)$.

Given $\varphi \in C^{1}(X)$ and $c \in \mathbb{R}$, we introduce the following sets:

$$
\varphi^{c}=\{u \in X: \varphi(u) \leqslant c\}, K_{\varphi}=\left\{u \in X: \varphi^{\prime}(u)=0\right\}, K_{\varphi}^{c}=\left\{u \in K_{\varphi}: \varphi(u)=c\right\} .
$$

Let $u \in X$ be an isolated critical point of $\varphi$ with $\varphi(u)=c$ (that is, $u \in K_{\varphi}^{c}$ ). Then the critical groups of $\varphi$ at $u$ are defined by

$$
C_{k}(\varphi, u)=H_{k}\left(\varphi^{c} \cap U, \varphi^{c} \cap U \backslash\{u\}\right) \text { for all } k \geqslant 0,
$$


where $U$ is a neighborhood of $u$ such that $K_{\varphi} \cap \varphi^{c} \cap U=\{u\}$. The excision property of the singular homology theory implies that the above definition of critical groups is independent of the particular choice of the neighborhood $U$.

Finally we mention that if $h: \Omega \times \mathbb{R} \rightarrow \mathbb{R}$ is a measurable function (for example, a Carathéodory function), we denote by $N_{h}$ the Nemytski operator corresponding to $h$, that is,

$$
N_{h}(u)(x)=h(\cdot, u(\cdot)) \text { for all } u \in H^{1}(\Omega) .
$$

\section{Solutions OF CONSTANT SigN}

In this section, we produce solutions of constant sign for problem $\left(P_{\lambda}\right)$ when $\lambda>0$ is sufficiently small. To do this, we introduce the following hypotheses on the data of the problem.

$H_{0}: \beta \in L^{s}(\Omega)$ with $s>N$.

$H_{1}: f: \Omega \times \mathbb{R} \rightarrow \mathbb{R}$ is a Carathéodory function such that $f(z, 0)=0$ for a.a. $z \in \Omega$ and

(i) $|f(z, x)| \leqslant a(z)(1+|x|)$ for a.a. $z \in \Omega$, all $x \in \mathbb{R}$ with $a \in L^{\infty}(\Omega)_{+}$;

(ii) $\limsup _{x \rightarrow \pm \infty} \frac{f(z, x)}{x} \leqslant \hat{\lambda}_{1}(\beta)$ uniformly for a.a. $z \in \Omega$;

(iii) $\lim _{x \rightarrow \pm \infty}[f(z, x) x-2 F(z, x)]=+\infty$ uniformly for a.a. $z \in \Omega$, where $F(z, x)=$ $\int_{0}^{x} f(z, \tau) d \tau$

(iv) there exist functions $\eta_{0}, \hat{\eta}_{0} \in L^{\infty}(\Omega)$ such that

$$
\begin{aligned}
& \hat{\lambda}_{1}(\beta) \leqslant \eta_{0}(z) \text { a.e. in } \Omega, \eta_{0} \neq \hat{\lambda}_{1}(\beta), \\
& \eta_{0}(z) \leqslant \liminf _{x \rightarrow 0} \frac{f(z, x)}{x} \leqslant \limsup _{x \rightarrow 0} \frac{f(z, x)}{x} \leqslant \hat{\eta}_{0}(z) \text { uniformly for a.a. } z \in \Omega .
\end{aligned}
$$

Remark 2. Hypothesis $H_{1}$ (ii) implies that asymptotically at $\pm \infty$ we can have resonance with respect to the principal eigenvalue $\hat{\lambda}_{1}(\beta)$. This justifies the presence of hypothesis $H_{1}$ (iii), which is needed in order for the energy functional to satisfy the PS-condition. Hypothesis $H_{1}$ (iv) implies that at the origin we have nonuniform nonresonance with respect to $\hat{\lambda}_{1}(\beta)$.

To produce solutions of constant sign, we introduce the following truncationsperturbations of the reaction of problem $\left(P_{\lambda}\right)$ :

$$
\begin{aligned}
& \hat{h}_{\lambda}^{+}(z, x)= \begin{cases}0 & \text { if } x \leqslant 0, \\
f(z, x)+\gamma x-\lambda x^{q-1} & \text { if } x>0 .\end{cases} \\
& \hat{h}_{\lambda}^{-}(z, x)= \begin{cases}f(z, x)+\gamma x-\lambda|x|^{q-2} x & \text { if } x<0, \\
0 & \text { if } x \geqslant 0 .\end{cases}
\end{aligned}
$$

Here $\gamma>0$ is as in inequality (4). Both $\hat{h}_{\lambda}^{ \pm}(z, x)$ are Carathéodory functions. We set $\hat{H}_{\lambda}^{ \pm}(z, x)=\int_{0}^{x} \hat{h}_{\lambda}^{ \pm}(z, \tau) d \tau$ and consider the $C^{1}$-functionals $\hat{\varphi}_{\lambda}^{ \pm}: H^{1}(\Omega) \rightarrow \mathbb{R}$ defined by

$$
\hat{\varphi}_{\lambda}^{ \pm}(u)=\frac{1}{2} \tau(u)+\frac{\gamma}{2}\|u\|_{2}^{2}-\int_{\Omega} \hat{H}_{\lambda}^{ \pm}(z, u(z)) d z \text { for all } u \in H^{1}(\Omega) .
$$


Also let $\varphi_{\lambda}: H^{1}(\Omega) \rightarrow \mathbb{R}$ be the energy functional for problem $\left(P_{\lambda}\right)$ defined by

$$
\varphi_{\lambda}(u)=\frac{1}{2} \tau(u)+\frac{\lambda}{q}\|u\|_{q}^{q}-\int_{\Omega} F(z, u(z)) d z \text { for all } u \in H^{1}(\Omega) .
$$

Evidently $\varphi_{\lambda} \in C^{1}\left(H^{1}(\Omega)\right)$.

Proposition 3. If hypotheses $H_{0}$ and $H_{1}$ hold and $\lambda>0$, then the functionals $\hat{\varphi}_{\lambda}^{ \pm}$ and $\varphi_{\lambda}$ are all coercive.

Proof. We do the proof for $\varphi_{\lambda}^{+}$, the proofs for $\varphi_{\lambda}^{-}$and $\varphi_{\lambda}$ being similar.

We argue indirectly. So, suppose that the functional $\varphi_{\lambda}^{+}$is not coercive. Then we can find $\left\{u_{n}\right\}_{n \geqslant 1} \subseteq H^{1}(\Omega)$ and $M_{1}>0$ such that

$$
\left\|u_{n}\right\| \rightarrow \infty \text { and } \hat{\varphi}_{\lambda}^{+}\left(u_{n}\right) \leqslant M_{1} \text { for all } n \geqslant 1 \text {. }
$$

So, we have

$$
\frac{1}{2} \tau\left(u_{n}\right)+\frac{\gamma}{2}\left\|u_{n}\right\|_{2}^{2}-\int_{\Omega} \hat{H}_{\lambda}^{+}\left(z, u_{n}\right) d z \leqslant M_{1} \text { for all } n \geqslant 1 .
$$

Let $y_{n}=\frac{u_{n}}{\left\|u_{n}\right\|}, n \geqslant 1$. Then $\left\|y_{n}\right\|=1$ for all $n \geqslant 1$, and so we may assume that

$$
y_{n} \stackrel{w}{\rightarrow} y \text { in } H^{1}(\Omega) \text { and } y_{n} \rightarrow y \text { in } L^{2}(\Omega) .
$$

From (7) and (8) we have

(10) $\frac{1}{2} \tau\left(y_{n}\right)+\frac{\gamma}{2}\left\|y_{n}^{-}\right\|_{2}^{2}-\int_{\Omega} \frac{F\left(z, u_{n}^{+}\right)}{\left\|u_{n}\right\|^{2}} d z+\frac{\lambda}{q} \frac{1}{\left\|u_{n}\right\|^{2-q}}\left\|y_{n}^{+}\right\|_{q}^{q} \leqslant \frac{M_{1}}{\left\|u_{n}\right\|^{2}}$ for all $n \geqslant 1$.

By virtue of hypothesis $H_{1}(\mathrm{i})$ we have that

$$
\left\{\frac{F\left(\cdot, u_{n}^{+}(\cdot)\right)}{\left\|u_{n}\right\|^{2}}\right\}_{n \geqslant 1} \subseteq L^{1}(\Omega) \text { is uniformly integrable. }
$$

So, by the Dunford-Pettis theorem and hypothesis $H_{1}$ (ii), we have (at least for a subsequence) that

$$
\frac{F\left(\cdot, u_{n}^{+}(\cdot)\right)}{\left\|u_{n}\right\|^{2}} \stackrel{w}{\rightarrow} \frac{1}{2} \vartheta\left(y^{+}\right)^{2} \text { in } L^{1}(\Omega),
$$

with $\vartheta \in L^{\infty}(\Omega)$ satisfying $\vartheta(z) \leqslant \hat{\lambda}_{1}(\beta)$ a.e. in $\Omega$ (see also Aizicovici, Papageorgiou and Staicu [1, proof of Proposition 14). Note that the functional $\tau(\cdot)$ is sequentially weakly lower semicontinuous (an easy consequence of the Sobolev embedding theorem). So, if in (10) we pass to the limit as $n \rightarrow \infty$ and use (91), (11) and the fact that $q<2$, we obtain

$$
\begin{aligned}
& \frac{1}{2} \tau(y)+\frac{\gamma}{2}\left\|y^{-}\right\|_{2}^{2} \leqslant \frac{1}{2} \int_{\Omega} \vartheta(z)\left(y^{+}\right)^{2} d z \\
\Rightarrow & \tau\left(y^{+}\right) \leqslant \int_{\Omega} \vartheta(z)\left(y^{+}\right)^{2} d z(\text { see (4) }) .
\end{aligned}
$$

If $\vartheta \neq \hat{\lambda}_{1}(\beta)$, then from (13) and Proposition 2, we have

$$
c_{0}\left\|y^{+}\right\|^{2} \leqslant 0 \text {, hence } y^{+}=0 \text {. }
$$

Also from (12) and (4), we have

$$
\hat{c}\left\|y^{-}\right\|^{2} \leqslant 0 \text {, hence } y^{-}=0 .
$$


Therefore $y=0$ and so we have

$$
\begin{aligned}
& \frac{\hat{H}_{\lambda}^{+}\left(\cdot, u_{n}(\cdot)\right)}{\left\|u_{n}\right\|^{2}} \stackrel{w}{\rightarrow} 0 \text { in } L^{1}(\Omega) \text { (as above) } \\
\Rightarrow & \tau\left(u_{n}\right)+\gamma\left\|u_{n}\right\|_{2}^{2} \rightarrow 0(\text { see (묘) }) \\
\Rightarrow & y_{n} \rightarrow 0 \text { in } H^{1}(\Omega)(\text { see (4) }),
\end{aligned}
$$

a contradiction to the fact that $\left\|y_{n}\right\|=1$ for all $n \geqslant 1$.

Next we assume that $\vartheta(z)=\hat{\lambda}_{1}(\beta)$ a.e. in $\Omega$ (resonant case). From (13) and (6), we infer that

$$
\begin{aligned}
& \tau\left(y^{+}\right)=\hat{\lambda}_{1}(\beta)\left\|y^{+}\right\|_{2}^{2} \\
\Rightarrow & y^{+}=\xi \hat{u}_{1}(\beta) \text { for some } \xi \geqslant 0 .
\end{aligned}
$$

First assume that $\xi=0$. Then $y^{+}=0$, and from (12) and (41) we also have $y^{-}=0$. Hence $y=0$. Then reasoning as above, we obtain that $y_{n} \rightarrow 0$ in $H^{1}(\Omega)$, a contradiction to the fact that $\left\|y_{n}\right\|=1$ for all $n \geqslant 1$.

So, suppose that $\xi>0$. Then $y^{+}(z)>0$ for all $z \in \Omega$ and so $y(z)=y^{+}(z)>0$ for all $z \in \Omega$, and we have

$$
u_{n}(z)=u_{n}^{+}(z) \rightarrow+\infty \text { for a.a. } z \in \Omega .
$$

For a.a. $z \in \Omega$ and all $x>0$, we have

$$
\frac{d}{d x}\left(\frac{F(z, x)}{x^{2}}\right)=\frac{f(z, x) x^{2}-2 x F(z, x)}{x^{4}}=\frac{f(z, x) x-2 F(z, x)}{x^{3}} .
$$

By virtue of hypothesis $H_{1}$ (iii), given $\xi>0$, we can find $M_{2}>0$ such that

$$
f(z, x) x-2 F(z, x) \geqslant \xi \text { for a.a. } z \in \Omega \text {, all } x \geqslant M_{2} .
$$

Using this in (15), we obtain

$$
\begin{aligned}
& \frac{d}{d x}\left(\frac{F(z, x)}{x^{2}}\right) \geqslant \frac{\xi}{x^{2}} \text { for a.a. } z \in \Omega, \text { all } x \geqslant M_{2} \\
\Rightarrow & \frac{F(z, u)}{u^{2}}-\frac{F(z, x)}{x^{2}} \geqslant-\frac{\xi}{2}\left[\frac{1}{u^{2}}-\frac{1}{x^{2}}\right] \text { for a.a. } z \in \Omega \text {, all } u \geqslant x \geqslant M_{2} .
\end{aligned}
$$

Passing to the limit as $u \rightarrow+\infty$ and using hypothesis $H_{1}($ ii), we obtain

$$
\begin{aligned}
& \frac{\hat{\lambda}_{1}(\beta)}{2}-\frac{F(z, x)}{x^{2}} \geqslant \frac{\xi}{2} \frac{1}{x^{2}} \text { for a.a. } z \in \Omega, \text { all } x \geqslant M_{2} \\
\Rightarrow \quad & \hat{\lambda}_{1}(\beta) x^{2}-2 F(z, x) \geqslant \xi \text { for a.a. } z \in \Omega, \text { all } x \geqslant M_{2} .
\end{aligned}
$$

Since $\xi>0$ is arbitrary, we infer that

(16) $\quad \hat{\lambda}_{1}(\beta) x^{2}-2 F(z, x) \rightarrow+\infty$ as $x \rightarrow+\infty$ uniformly for a.a. $z \in \Omega$.

From (8), (7) and (6), we have

$$
0 \leqslant \frac{\gamma}{2}\left\|u_{n}^{-}\right\|_{2}^{2} \leqslant M_{1}-\int_{\Omega}\left[\hat{\lambda}_{1}(\beta)\left(u_{n}^{+}\right)^{2}-2 F\left(z, u_{n}^{+}\right)\right] d z .
$$

From (14), (16) and Fatou's lemma, it follows that

$$
\int_{\Omega}\left[\hat{\lambda}_{1}(\beta)\left(u_{n}^{+}\right)^{2}-2 F\left(z, u_{n}^{+}\right)\right] d z \rightarrow+\infty .
$$

Comparing (17) and (18), we reach a contradiction. This proves the coercivity of $\hat{\varphi}_{\lambda}^{+}$. In a similar fashion, we show the coercivity of $\hat{\varphi}_{\lambda}^{-}$and of $\varphi_{\lambda}$. 
From the above proposition, the following result concerning the compactness properties of the functionals $\hat{\varphi}_{\lambda}^{ \pm}$and $\varphi_{\lambda}$ follows easily.

Corollary 4. If hypotheses $H_{0}$ and $H_{1}$ hold and $\lambda>0$, then the functionals $\hat{\varphi}_{\lambda}^{ \pm}$ and $\varphi_{\lambda}$ satisfy the PS-condition.

Proposition 3 permits the use of the direct method on the functionals $\hat{\varphi}_{\lambda}^{ \pm}$. The next result guarantees that for small $\lambda>0$ the minimizers of $\hat{\varphi}_{\lambda}^{ \pm}$obtained by the direct method are nontrivial.

Proposition 5. If hypotheses $H_{0}$ and $H_{1}$ hold, then we can find $\lambda^{*}>0$ such that for every $\lambda \in\left(0, \lambda^{*}\right)$, there exists $t^{*}=t^{*}(\lambda)>0$ for which we have

$$
\varphi_{\lambda}\left( \pm t^{*} \hat{u}_{1}(\beta)\right)<0 \text {. }
$$

Proof. By virtue of hypotheses $H_{1}(\mathrm{i})$, (iv), given $\varepsilon>0$, we can find $c_{1}=c_{1}(\varepsilon)>0$ such that

$$
F(z, x) \geqslant \frac{1}{2}\left(\eta_{0}(z)-\varepsilon\right) x^{2}-c_{1}|x|^{r} \text { for a.a. } z \in \Omega, \text { all } x \in \mathbb{R}, \text { with } r>2 .
$$

For $t \neq 0$, we have

$$
\begin{aligned}
& \varphi_{\lambda}\left(t \hat{u}_{1}(\beta)\right)=\frac{t^{2}}{2} \tau\left(\hat{u}_{1}(\beta)\right)+\frac{|t|^{q} \lambda}{q}\left\|\hat{u}_{1}(\beta)\right\|_{q}^{q}-\int_{\Omega} F\left(z, t \hat{u}_{1}(\beta)\right) d z \\
& \leqslant \frac{t^{2}}{2}\left[\tau\left(\hat{u}_{1}(\beta)\right)-\int_{\Omega}\left(\eta_{0}(z)-\varepsilon\right) \hat{u}_{1}(\beta)^{2} d z\right] \\
& \quad+c_{1}|t|^{r}\left\|\hat{u}_{1}(\beta)\right\|_{r}^{r}+\frac{|t|^{q} \lambda}{q}\left\|\hat{u}_{1}(\beta)\right\|_{q}^{q}(\text { see (18) })
\end{aligned}
$$

We know that $\hat{u}_{1}(\beta)(z)>0$ for all $z \in \Omega$. So

$$
\xi^{*}=\int_{\Omega}\left(\eta_{0}(z)-\hat{\lambda}_{1}(\beta)\right) \hat{u}_{1}(\beta)^{2} d z>0\left(\text { see } H_{1}(\mathrm{iv})\right) .
$$

Choosing $\varepsilon \in\left(0, \xi^{*}\right)$, we obtain

$$
\begin{aligned}
\varphi_{\lambda}\left(t \hat{u}_{1}(\beta)\right) & \leqslant-c_{2} t^{2}+c_{3}\left(|t|^{r}+\frac{\lambda}{q}|t|^{q}\right) \text { for some } c_{2}, c_{3}>0, \text { all } t \neq 0 \\
& =\left[-c_{2}+c_{3}\left(|t|^{r-2}+\frac{\lambda}{q}|t|^{q-2}\right)\right] t^{2} \text { for all } t \neq 0 .
\end{aligned}
$$

Let $\mu_{\lambda}(\tau)=\tau^{r-2}+\frac{\lambda}{q} \tau^{q-2}$ for all $\tau>0$. Evidently $\mu_{\lambda} \in C^{1}(0,+\infty)$, and since $q<2<r$, we see that

$$
\mu_{\lambda}(\tau) \rightarrow+\infty \text { as } \tau \rightarrow 0^{+} \text {and as } \tau \rightarrow+\infty
$$


So, we can find $\tau_{0}>0$ such that

$$
\begin{aligned}
& \mu_{\lambda}\left(\tau_{0}\right)=\inf [\mu(\tau): \tau>0]>0 \\
\Rightarrow & \mu_{\lambda}^{\prime}\left(\tau_{0}\right)=0 \\
\Rightarrow & \frac{\lambda}{q}(2-q) \tau_{0}^{q-3}=(r-2) \tau_{0}^{r-3} \\
\Rightarrow & \tau_{0}=\tau_{0}(\lambda)=\left[\frac{\lambda(2-q)}{q(r-2)}\right]^{\frac{1}{r-q}} .
\end{aligned}
$$

Then $\mu_{\lambda}\left(\tau_{0}(\lambda)\right) \rightarrow 0^{+}$as $\lambda \rightarrow 0^{+}$. So, we can find $\lambda^{*}>0$ such that

$$
\mu_{\lambda}\left(\tau_{0}\right)<\frac{c_{2}}{c_{3}} \text { for all } \lambda \in\left(0, \lambda^{*}\right) .
$$

Taking $t^{*}=\tau_{0}(\lambda)$, from (20) we have

$$
\varphi_{\lambda}\left( \pm t^{*} \hat{u}_{1}(\beta)\right)<0 .
$$

The next proposition will be useful in applying the mountain pass theorem.

Proposition 6. If hypotheses $H_{0}$ and $H_{1}$ hold and $\lambda>0$, then $u=0$ is a local minimizer for the functionals $\hat{\varphi}_{\lambda}^{ \pm}$and $\varphi_{\lambda}$.

Proof. Again we do the proof for the functional $\hat{\varphi}_{\lambda}^{+}$, the proofs for the functionals $\hat{\varphi}_{\lambda}^{ \pm}$and $\varphi_{\lambda}$ being similar.

By virtue of hypotheses $H_{1}(\mathrm{i})$, (iv), we see that there exists $c_{4}>0$ such that

$$
|F(z, x)| \leqslant c_{4} x^{2} \text { for a.a. } z \in \Omega \text {, all } x \in \mathbb{R} \text {. }
$$

Then for every $u \in H^{1}(\Omega)$, we have

$$
\begin{aligned}
\hat{\varphi}_{\lambda}^{+}(u) & =\frac{1}{2} \tau(u)+\frac{\gamma}{2}\|u\|_{2}^{2}-\int_{\Omega} \hat{H}_{\lambda}^{+}(z, u) d z \\
& =\frac{1}{2} \tau(u)+\frac{\gamma}{2}\left\|u^{-}\right\|_{2}^{2}+\frac{\lambda}{q}\left\|u^{+}\right\|_{q}^{q}-\int_{\Omega} F\left(z, u^{+}\right) d z \text { (see (17)) } \\
& \geqslant \frac{1}{2} \tau\left(u^{+}\right)+\frac{\lambda}{q}\left\|u^{+}\right\|_{q}^{q}-c_{4}\left\|u^{+}\right\|_{2}^{2}+\frac{1}{2} \tau\left(u^{-}\right)+\frac{\gamma}{2}\left\|u^{-}\right\|_{2}^{2} \text { (see (21)) } \\
& \left.\geqslant \frac{\hat{c}}{2}\left\|u^{+}\right\|^{2}-\frac{\gamma}{2}\left\|u^{+}\right\|_{2}^{2}+\frac{\lambda}{q}\left\|u^{+}\right\|_{q}^{q}-c_{4}\left\|u^{+}\right\|_{2}^{2} \text { (see (4) ) }\right) \\
& \geqslant \int_{\Omega}\left[\frac{\lambda}{q}\left(u^{+}\right)^{q}-c_{5}\left(u^{+}\right)^{2}\right] d z \text { with } c_{5}=c_{4}+\frac{\gamma}{2}>0 .
\end{aligned}
$$

Suppose that we could find $\left\{u_{n}\right\}_{n \geqslant 1} \subseteq C^{1}(\bar{\Omega})$ such that $u_{n} \rightarrow 0$ in $C^{1}(\bar{\Omega})$ and

$$
\begin{aligned}
& \left.\hat{\varphi}_{\lambda}^{+}\left(u_{n}\right)<0, \text { hence } u_{n}^{+} \neq 0 \text { for all } n \geqslant 1 \text { (see (7) }\right) \\
\Rightarrow & \left.\int_{\Omega}\left[\frac{\lambda}{q}-c_{5}\left\|u_{n}^{+}\right\|_{\infty}^{2-q}\right]\left(u_{n}^{+}\right)^{q} d z<0 \text { for all } n \geqslant 1 \text { (see (22) }\right) .
\end{aligned}
$$

Since $u_{n} \rightarrow 0$ in $C^{1}(\bar{\Omega})$, it follows that $u_{n}^{+} \rightarrow 0$ in $C(\bar{\Omega})$. So, we can find $n_{0} \in \mathbb{N}$ such that

$$
\int_{\Omega}\left[\frac{\lambda}{q}-c_{5}\left\|u_{n}^{+}\right\|_{\infty}^{2-q}\right]\left(u_{n}^{+}\right)^{q} d z>0 \text { for all } n \geqslant n_{0}
$$


Evidently, relations (23) and (24) lead to a contradiction. This proves that $u=0$ is a local $C^{1}(\bar{\Omega})$-minimizer of $\hat{\varphi}_{\lambda}^{+}$. Invoking Proposition 11, we conclude that $u=0$ is a local $H^{1}(\Omega)$-minimizer of $\hat{\varphi}_{\lambda}^{+}$.

Similarly for the functionals $\hat{\varphi}_{\lambda}^{-}$and $\varphi_{\lambda}$.

Now we are ready to produce constant sign solutions for problem $\left(P_{\lambda}\right)$ when $\lambda>0$ is small, namely, when $\lambda \in\left(0, \lambda^{*}\right)$.

Proposition 7. If hypotheses $H_{0}$ and $H_{1}$ hold and $\lambda \in\left(0, \lambda^{*}\right)$, then problem $\left(P_{\lambda}\right)$ has at least four nontrivial constant sign solutions:

$$
\begin{gathered}
u_{0}, \hat{u} \in C_{+} \text {with } u_{0}(z), \hat{u}(z)>0 \text { for all } z \in \Omega, \\
v_{0}, \hat{v} \in-C_{+} \text {with } v_{0}(z), \hat{v}(z)<0 \text { for all } z \in \Omega .
\end{gathered}
$$

Moreover, $u_{0}, v_{0}$ are local minimizers of the energy functional $\varphi_{\lambda}$ while $\hat{u}, \hat{v}$ have positive energy, that is, $\varphi_{\lambda}(\hat{u}), \varphi_{\lambda}(\hat{v})>0$.

Proof. By virtue of Proposition 3, the functional $\hat{\varphi}_{\lambda}^{+}$is coercive. Also, using the Sobolev embedding theorem, we can see that $\hat{\varphi}_{\lambda}^{+}$is sequentially weakly lower semicontinuous. So, by the Weierstrass theorem, we can find $u_{0} \in H^{1}(\Omega)$ such that

$$
\hat{\varphi}_{\lambda}^{+}\left(u_{0}\right)=\inf \left[\hat{\varphi}_{\lambda}^{+}(u): u \in H^{1}(\Omega)\right] .
$$

Since $\lambda \in\left(0, \lambda^{*}\right)$, from Proposition 5 we know that

$$
\begin{aligned}
& \hat{\varphi}_{\lambda}^{+}\left(t^{*} \hat{u}_{1}(\beta)\right)<0 \\
\Rightarrow \quad & \hat{\varphi}_{\lambda}^{+}\left(u_{0}\right)<0=\hat{\varphi}_{\lambda}^{+}(0)(\text { see }(25)) \text {, hence } u_{0} \neq 0 .
\end{aligned}
$$

From (25) we have

$$
\begin{aligned}
& \left(\hat{\varphi}_{\lambda}^{+}\right)^{\prime}\left(u_{0}\right)=0 \\
\Rightarrow \quad & A\left(u_{0}\right)+(\beta(z)+\gamma) u_{0}=N_{\hat{h}_{\lambda}^{+}}\left(u_{0}\right),
\end{aligned}
$$

where $A \in \mathcal{L}\left(H^{1}(\Omega), H^{1}(\Omega)^{*}\right)$ is defined by

$$
\langle A(u), y\rangle=\int_{\Omega}(D u, D y)_{\mathbb{R}^{N}} d z \text { for all } u, y \in H^{1}(\Omega) .
$$

On (26) we act with $-u_{0}^{-} \in H^{1}(\Omega)$. We obtain

$$
\begin{aligned}
& \tau\left(u_{0}^{-}\right)+\gamma\left\|u_{0}^{-}\right\|_{2}^{2}=0(\text { see (17) }) \\
\Rightarrow \quad & \hat{c}\left\|u_{0}^{-}\right\|^{2} \leqslant 0(\text { see (4) }) \text {, hence } u_{0} \geqslant 0, u_{0} \neq 0 .
\end{aligned}
$$

Then (26) becomes

$$
\begin{aligned}
& A\left(u_{0}\right)+\beta(z) u_{0}=N_{f}\left(u_{0}\right)-\lambda u_{0}^{q-1}(\text { see }(7)) \\
\Rightarrow & -\Delta u_{0}(z)+\beta(z) u_{0}(z)=f\left(z, u_{0}(z)\right)-\lambda u_{0}(z)^{q-1} \text { a.e. in } \Omega, \\
& \frac{\partial u_{0}}{\partial n}=0 \text { on } \partial \Omega \text { (see Motreanu and Papageorgiou [7]). }
\end{aligned}
$$

Using Lemmata 5.1 and 5.2 of Wang [15], as in Papageorgiou and Rădulescu [1] (see the proof of Proposition 3.2, p. 303), we have that $u_{0} \in C_{+} \backslash\{0\}$. Harnack's inequality (see Pucci and Serrin [14, p. 163]) implies that

$$
u_{0}(z)>0 \text { for all } z \in \Omega \text {. }
$$


Claim 1. $u_{0}$ is a local minimizer of $\varphi_{\lambda}$.

According to Proposition 1, it suffices to show that $u_{0}$ is a local $C^{1}(\bar{\Omega})$-minimizer of $\varphi_{\lambda}$. We argue by contradiction. So, suppose that $u_{0}$ is not a local $C^{1}(\bar{\Omega})$ minimizer of $\varphi_{\lambda}$. Then we can find $\left\{u_{n}\right\}_{n \geqslant 1} \subseteq C^{1}(\bar{\Omega})$ such that

$$
u_{n} \rightarrow u_{0} \text { in } C^{1}(\bar{\Omega}) \text { and } \varphi_{\lambda}\left(u_{n}\right)<\varphi_{\lambda}\left(u_{0}\right) \text { for all } n \geqslant 1 \text {. }
$$

From (77) it is clear that $\left.\varphi_{\lambda}\right|_{C_{+}}=\left.\hat{\varphi}_{\lambda}^{+}\right|_{C_{+}}$. So, we have

$$
\begin{aligned}
0 \geqslant & \varphi_{\lambda}\left(u_{n}\right)-\varphi_{\lambda}\left(u_{0}\right) \\
= & \varphi_{\lambda}\left(u_{n}\right)-\hat{\varphi}_{\lambda}^{+}\left(u_{0}\right) \\
\geqslant & \varphi_{\lambda}\left(u_{n}\right)-\hat{\varphi}_{\lambda}^{+}\left(u_{n}\right)(\text { see }(\underline{25})) \\
= & \frac{1}{2} \tau\left(u_{n}\right)+\frac{\lambda}{q}\left\|u_{n}\right\|_{q}^{q}-\int_{\Omega} F\left(z, u_{n}\right) d z \\
& \left.-\frac{1}{2} \tau\left(u_{n}\right)-\frac{\gamma}{2}\left\|u_{n}^{-}\right\|_{2}^{2}-\frac{\lambda}{q}\left\|u_{n}^{+}\right\|_{q}^{q}+\int_{\Omega} F\left(z, u_{n}^{+}\right) d z \text { (see (7)) }\right) \\
= & \frac{\lambda}{q}\left\|u_{n}^{-}\right\|_{q}^{q}-\frac{\gamma}{2}\left\|u_{n}^{-}\right\|_{2}^{2}-\int_{\Omega} F\left(z,-u_{n}^{-}\right) d z \\
\geqslant & \frac{\lambda}{q}\left\|u_{n}^{-}\right\|_{q}^{q}-\frac{\gamma}{2}\left\|u_{n}^{-}\right\|_{2}^{2}-c_{4}\left\|u_{n}^{-}\right\|_{2}^{2}(\operatorname{see}(\underline{21})) \\
\geqslant & \int_{\Omega}\left[\frac{\lambda}{q}-\left(\frac{\gamma}{2}+c_{4}\right)\left\|u_{n}^{-}\right\|_{\infty}^{2-q}\right]\left(u_{n}^{-}\right)^{q} d z .
\end{aligned}
$$

Because $u_{0}(z)>0$ for all $z \in \Omega$ and $u_{n} \rightarrow u_{0}$ in $C^{1}(\bar{\Omega})$, it follows that

$$
u_{n}^{-} \rightarrow 0 \text { in } C(\bar{\Omega}) \text {. }
$$

So, from (28) we see that we can find $n_{0} \in \mathbb{N}$ such that

$$
0>\int_{\Omega}\left[\frac{\lambda}{q}-\left(\frac{\gamma}{2}+c_{4}\right)\left\|u_{n}^{-}\right\|_{\infty}^{2-q}\right]\left(u_{n}^{-}\right)^{q} d z \geqslant 0 \text { for all } n \geqslant n_{0},
$$

a contradiction. This proves Claim 1 .

From Proposition [6 we know that $u=0$ is a local minimizer of $\varphi_{\lambda}$, too. Then as in Aizicovici, Papageorgiou and Staicu [1] (see the proof of Proposition 29), we can find $\varrho \in(0,1)$ small such that

$$
\hat{\varphi}_{\lambda}^{+}\left(u_{0}\right)<0=\hat{\varphi}_{\lambda}^{+}(0)<\inf \left[\hat{\varphi}_{\lambda}^{+}(u):\|u\|=\varrho\right]=\hat{m}_{\lambda, \varrho}^{+} .
$$

Then (29) and Corollary 4 permit the use of the mountain pass theorem. So, we can find $\hat{u} \in H^{1}(\Omega)$ such that

$$
\hat{u} \in K_{\hat{\varphi}_{\lambda}^{+}} \text {and } 0=\hat{\varphi}_{\lambda}^{+}(0)<\hat{m}_{\lambda, \varrho}^{+} \leqslant \hat{\varphi}_{\lambda}^{+}(\hat{u}) .
$$

From (29) and (30) it follows that $\hat{u} \notin\left\{0, u_{0}\right\}, \hat{u} \in C_{+} \backslash\{0\}$, solves $\left(P_{\lambda}\right)$ (this is established as we did for $\left.u_{0}\right)$, and via Harnack's inequality we have $\hat{u}(z)>0$ for all $z \in \Omega$.

Similarly, working with $\hat{\varphi}_{\lambda}^{-}$, we produce $v_{0}, \hat{v} \in\left(-C_{+}\right) \backslash\{0\}$ solutions of $\left(P_{\lambda}\right)$ such that

$$
v_{0}(z), \hat{v}(z)<0 \text { for all } z \in \Omega
$$

with $v_{0}$ a local minimizer of $\varphi_{\lambda}$ and $0<\varphi_{\lambda}(\hat{v})$. 


\section{Five solutions}

To produce a fifth solution (but without any sign information), we need to restrict the behavior of the perturbation $f(z, \cdot)$ near the origin. So, the new hypotheses on $f(z, x)$ are the following:

$H_{2}: f: \Omega \times \mathbb{R} \rightarrow \mathbb{R}$ is a Carathéodory function such that $f(z, 0)=0$ for a.a. $z \in \Omega$ and

(i) $|f(z, x)| \leqslant a(z)(1+|x|)$ for a.a. $z \in \Omega$, all $x \in \mathbb{R}$ with $a \in L^{\infty}(\Omega)_{+}$;

(ii) $\limsup _{x \rightarrow \pm \infty} \frac{f(z, x)}{x} \leqslant \hat{\lambda}_{1}(\beta)$ uniformly for a.a. $z \in \Omega$;

(iii) $\lim _{x \rightarrow+\infty}[f(z, x) x-2 F(z, x)]=+\infty$ uniformly for a.a. $z \in \Omega$;

(iv) there exist an integer $m \geqslant 2$ and functions $\eta_{0}, \hat{\eta}_{0} \in L^{\infty}(\Omega)$ such that

$$
\begin{aligned}
& \hat{\lambda}_{m}(\beta) \leqslant \eta_{0}(z) \leqslant \hat{\eta}_{0}(z) \leqslant \hat{\lambda}_{m+1}(\beta) \text { a.e. in } \Omega, \hat{\lambda}_{m}(\beta) \neq \eta_{0}, \hat{\lambda}_{m+1}(\beta) \neq \hat{\eta}_{0}, \\
& \eta_{0}(z) \leqslant \liminf _{x \rightarrow 0} \frac{f(z, x)}{x} \leqslant \limsup _{x \rightarrow 0} \frac{f(z, x)}{x} \leqslant \hat{\eta}_{0}(z) \text { uniformly for a.a. } z \in \Omega, \\
& F(z, x) \leqslant \frac{\hat{\lambda}_{m+1}(\beta)}{2} x^{2} \text { for a.a. } z \in \Omega, \text { all } x \in \mathbb{R} .
\end{aligned}
$$

Let $Y=\bigoplus_{k=1}^{m} E\left(\hat{\lambda}_{k}(\beta)\right)$ and $V=\overline{\bigoplus_{k \geqslant m+1} E\left(\hat{\lambda}_{k}(\beta)\right)}$. We have the orthogonal direct sum decomposition

$$
H^{1}(\Omega)=Y \oplus V
$$

We check the behavior of $\varphi_{\lambda}$ with respect to this decomposition.

Proposition 8. If hypotheses $H_{0}$ and $H_{2}$ hold, then we can find $\hat{\lambda}^{*} \in\left(0, \lambda^{*}\right]$ such that for all $\lambda \in\left(0, \hat{\lambda}^{*}\right)$ there exists $\varrho=\varrho(\lambda)>0$ for which we have

$$
\left.\varphi_{\lambda}\right|_{\partial B_{\varrho} \cap Y}<0
$$

(here $\lambda^{*}>0$ is as in Proposition 5 and $\partial B_{\varrho}=\left\{u \in H^{1}(\Omega):\|u\|=\varrho\right\}$ ).

Proof. By virtue of hypotheses $H_{2}(\mathrm{i})$, (iv), given $\varepsilon>0$, we can find $c_{6}=c_{6}(\varepsilon)>0$ such that

$$
F(z, x) \geqslant \frac{1}{2}\left(\eta_{0}(z)-\varepsilon\right) x^{2}-c_{6}|x|^{r} \text { for a.a. } z \in \Omega, \text { all } x \in \mathbb{R} \text { with } r>2 .
$$

For all $u \in Y$, we have

$$
\begin{aligned}
\varphi_{\lambda}(u)= & \frac{1}{2} \tau(u)+\frac{\lambda}{q}\|u\|_{q}^{q}-\int_{\Omega} F(z, u) d z \\
\leqslant & \frac{1}{2}\left[\tau(u)-\int_{\Omega} \eta_{0}(z) u^{2} d z+\varepsilon\|u\|^{2}\right]+c_{7}\left(\|u\|^{r}+\frac{\lambda}{q}\|u\|^{q}\right) \\
& \left(\text { for some } c_{7}>0(\text { see (131) })\right) \\
\leqslant & \frac{1}{2}\left[-\hat{\xi}_{0}+\varepsilon\right]\|u\|^{2}+c_{7}\left(\|u\|^{r}+\frac{\lambda}{q}\|u\|^{q}\right) \text { (see Proposition 22). }
\end{aligned}
$$

Choosing $\varepsilon \in\left(0, \hat{\xi}_{0}\right)$, we have

$$
\varphi_{\lambda}(u) \leqslant\left[-c_{8}+c_{7}\left(\|u\|^{r-2}+\frac{\lambda}{q}\|u\|^{q-2}\right)\right]\|u\|^{2} \text { for some } c_{8}>0 .
$$


As in the proof of Proposition 5, by considering the function $\mu_{\lambda}(\tau)=\tau^{r-2}+\frac{\lambda}{q} \tau^{q-2}$, we can find $\tilde{\lambda}^{*}>0$ such that for all $\lambda \in\left(0, \tilde{\lambda}^{*}\right)$ there exists $\varrho=\varrho(\lambda)>0$ for which we have

$$
\varphi_{\lambda}(u)<0 \text { for all } u \in \partial B_{\varrho} \cap Y .
$$

Finally let $\hat{\lambda}^{*}=\min \left\{\tilde{\lambda}^{*}, \lambda^{*}\right\}$.

Proposition 9. If hypotheses $H_{0}$ and $H_{2}$ hold, then $\left.\varphi_{\lambda}\right|_{V} \geqslant 0$.

Proof. Using hypothesis $H_{2}($ iv), for every $u \in V$, we have

$$
\varphi_{\lambda}(u) \geqslant \frac{1}{2} \tau(u)-\frac{\hat{\lambda}_{m+1}(\beta)}{2}\|u\|^{2} \geqslant 0 .
$$

Now we can produce a fifth nontrivial solution.

Proposition 10. If hypotheses $H_{0}$ and $H_{2}$ hold and $\lambda \in\left(0, \hat{\lambda}^{*}\right)$, then problem $\left(P_{\lambda}\right)$ has a fifth nontrivial solution: $y_{0} \in C^{1}(\bar{\Omega})$.

Proof. Proposition 3 implies that the energy functional $\varphi_{\lambda}$ is bounded below. This fact together with Propositions 8 and 9 permits the use of Theorem 3.1 of Perera [13. So, we can find $y_{0} \in H^{1}(\Omega)$ such that

$$
\begin{gathered}
y_{0} \in K_{\varphi_{\lambda}}, \varphi_{\lambda}\left(y_{0}\right)<0=\varphi_{\lambda}(0) \text { and } \\
C_{d_{m}-1}\left(\varphi_{\lambda}, 0\right) \neq 0\left(d_{m}=\operatorname{dim} \oplus_{k=1}^{m} E\left(\hat{\lambda}_{k}(\beta)\right) \geqslant 2\right) .
\end{gathered}
$$

Since $\varphi_{\lambda}(\hat{u}), \varphi_{\lambda}(\hat{v})>0$ (see Proposition 17), we have that $y_{0} \notin\{a, \hat{u}, \hat{v}\}$ (see also (32) ). From Proposition 7 we know that $u_{0}, v_{0}$ are local minimizers of $\varphi_{\lambda}$. Hence

$$
C_{k}\left(\varphi_{\lambda}, u_{0}\right)=C_{k}\left(\varphi_{\lambda}, v_{0}\right)=\delta_{k, 0} \mathbb{Z} \text { for all } k \geqslant 0 .
$$

Comparing (32) and (33), we see that $y_{0} \notin\left\{u_{0}, v_{0}\right\}$. Therefore $y_{0}$ is the fifth nontrivial solution of $\left(P_{\lambda}\right)$ (since $y_{0} \in K_{\varphi_{\lambda}}$; see (32)), and as before using the regularity result of Wang [15, we have $y_{0} \in C^{1}(\bar{\Omega})$.

So, we can state the following multiplicity theorem for problem $\left(P_{\lambda}\right)$.

Theorem 11. If hypotheses $H_{0}$ and $H_{2}$ hold, then there exists $\hat{\lambda}^{*}>0$ such that for all $\lambda \in\left(0, \hat{\lambda}^{*}\right)$ problem $\left(P_{\lambda}\right)$ has at least five nontrivial solutions:

$$
\begin{aligned}
& u_{0}, \hat{u} \in C_{+}, u_{0}(z), \hat{u}(z)>0 \text { for all } z \in \Omega, \\
& v_{0}, \hat{v} \in-C_{+}, v_{0}(z), \hat{v}(z)<0 \text { for all } z \in \Omega, \\
& y_{0} \in C^{1}(\bar{\Omega}) \backslash\{0\},
\end{aligned}
$$

with $u_{0}, v_{0}$ local minimizers of the energy functional and

$$
\varphi_{\lambda}\left(u_{0}\right), \varphi_{\lambda}\left(v_{0}\right), \varphi_{\lambda}\left(y_{0}\right)<0=\varphi_{\lambda}(0)<\varphi_{\lambda}(\hat{u}), \varphi_{\lambda}(\hat{v}) .
$$

Remark 3. It is an interesting open question whether the fifth solution $y_{0}$ is nodal (that is, sign changing). 


\section{REFERENCES}

[1] Sergiu Aizicovici, Nikolaos S. Papageorgiou, and Vasile Staicu, Degree theory for operators of monotone type and nonlinear elliptic equations with inequality constraints, Mem. Amer. Math. Soc. 196 (2008), no. 915, vi+70, DOI 10.1090/memo/0915. MR2459421(2010c:47211)

[2] Antonio Ambrosetti, Haïm Brezis, and Giovanna Cerami, Combined effects of concave and convex nonlinearities in some elliptic problems, J. Funct. Anal. 122 (1994), no. 2, 519-543, DOI 10.1006/jfan.1994.1078. MR1276168 (95g:35059)

[3] Antonio Ambrosetti and Paul H. Rabinowitz, Dual variational methods in critical point theory and applications, J. Functional Analysis 14 (1973), 349-381. MR0370183 (51 \#6412)

[4] Haïm Brezis and Louis Nirenberg, $H^{1}$ versus $C^{1}$ local minimizers (English, with English and French summaries), C. R. Acad. Sci. Paris Sér. I Math. 317 (1993), no. 5, 465-472. MR $1239032(94 \mathrm{~g}: 49044)$

[5] Francisco Odair de Paiva and Eugenio Massa, Multiple solutions for some elliptic equations with a nonlinearity concave at the origin, Nonlinear Anal. 66 (2007), no. 12, 2940-2946, DOI 10.1016/j.na.2006.04.015. MR2311647 (2008k:35171)

[6] Leszek Gasiński and Nikolaos S. Papageorgiou, Pairs of nontrivial solutions for resonant Neumann problems, J. Math. Anal. Appl. 398 (2013), no. 2, 649-663, DOI 10.1016/j.jmaa.2012.09.034. MR2990090

[7] Dumitru Motreanu and Nikolaos S. Papageorgiou, Existence and multiplicity of solutions for Neumann problems, J. Differential Equations 232 (2007), no. 1, 1-35, DOI 10.1016/j.jde.2006.09.008. MR2281188(2007h:35046)

[8] D. Motreanu and N. S. Papageorgiou, Multiple solutions for nonlinear Neumann problems driven by a nonhomogeneous differential operator, Proc. Amer. Math. Soc. 139 (2011), no. 10, 3527-3535, DOI 10.1090/S0002-9939-2011-10884-0. MR 2813384 (2012c:35166)

[9] Dimitri Mugnai and Nikolaos S. Papageorgiou, Resonant nonlinear Neumann problems with indefinite weight, Ann. Sc. Norm. Super. Pisa Cl. Sci. (5) 11 (2012), no. 4, 729-788. MR3060699

[10] Nikolaos S. Papageorgiou and Sophia Th. Kyritsi-Yiallourou, Handbook of applied analysis, Advances in Mechanics and Mathematics, vol. 19, Springer, New York, 2009. MR2527754 (2010g:49001)

[11] Nikolaos S. Papageorgiou and Vicenţiu D. Rădulescu, Semilinear Neumann problems with indefinite and unbounded potential and crossing nonlinearity, Recent trends in nonlinear partial differential equations. II. Stationary problems, Contemp. Math., vol. 595, Amer. Math. Soc., Providence, RI, 2013, pp. 293-315, DOI 10.1090/conm/595/11801. MR3156380

[12] N. S. Papageorgiou and V. D. Rădulescu, Coercive and noncoercive nonlinear Neumann problems with an indefinite potential, submitted.

[13] Kanishka Perera, Multiplicity results for some elliptic problems with concave nonlinearities, J. Differential Equations 140 (1997), no. 1, 133-141, DOI 10.1006/jdeq.1997.3310. MR 1473857 (99e:35070)

[14] Patrizia Pucci and James Serrin, The maximum principle, Progress in Nonlinear Differential Equations and their Applications, 73, Birkhäuser Verlag, Basel, 2007. MR2356201 (2008m:35001)

[15] Xu Jia Wang, Neumann problems of semilinear elliptic equations involving critical Sobolev exponents, J. Differential Equations 93 (1991), no. 2, 283-310, DOI 10.1016/00220396(91)90014-Z. MR.1125221 (92j:35072)

Department of Mathematics, National Technical University, Zografou Campus, Athens 15780, Greece

E-mail address: npapg@math.ntua.gr

Department of Mathematics, Faculty of Sciences, King Abdulaziz University, P.O. Box 80302, Jeddah 21589, Saudi Arabia - and - Institute of Mathematics "Simion Stoilow" of the Romanian Academy, P.O. Box 1-764, 014700 Bucharest, Romania

E-mail address: vicentiu.radulescu@math.cnrs.fr 